ISSN 1112-9867

http://www.jfas.info

\title{
GRADIENT REMEDIABILITY IN LINEAR DISTRIBUTED PARABOLIC SYSTEMS ANALYSIS, APPROXIMATIONS AND SIMULATIONS
}

\author{
S. Rekkab ${ }^{*}$ and S. Benhadid
}

Mathematics Department, Faculty of Exact Sciences, University Mentouri, Constantine, Algeria

Received: 28 April 2017 / Accepted: 22 August 2017 / Published online: 01 September 2017

\begin{abstract}
The aim of this paper is the introduction of a new concept that concerned the analysis of a large class of distributed parabolic systems. It is the general concept of gradient remediability. More precisely, we study with respect to the gradient observation, the existence of an input operator (gradient efficient actuators) ensuring the compensation of known or unknown disturbances acting on the considered system. Then, we introduce and we characterize the notions of exact and weak gradient remediability and their relationship with the notions of exact and weak gradient controllability. Main properties concerning the notion of gradient efficient actuators are considered. The minimum energy problem is studies, and we show how to find the optimal control, which compensates the disturbance of the system. Approximations and numerical simulations are also presented.
\end{abstract}

Keywords: actuators efficient; disturbance; gradient; parabolic systems; remediability; sensors.

Author Correspondence, e-mail: rekkabsoraya@yahoo.fr

doi: http://dx.doi.org/10.4314/jfas.v9i3.18 


\section{INTRODUCTION}

Various real problems can be formulated within certain concepts of distributed systems analysis. These concepts consist of a set of notions as controllability, detectability, observability, remediability..., which enable a better knowledge and understanding of the system to be obtained. These concepts have been studies at different degrees (exact, weak, extended). Systems analysis can be done from a purely theoretical viewpoint $[1,2]$. However, the study may be also become concrete, in some sense, by using the structures of actuators and sensors. Thus, one can study the different concepts of controllability via actuators structures [3-5] or the different concepts of observability via sensors structures [6-8].

An extension of these concepts that is very important in practical applications is that of gradient controllability [9], gradient detectability [10] and gradient observability [11-13]. These concepts are of great interest from a more practical and control point of view since there exist systems that cannot be controllable but gradient controllable or that cannot be observable but gradient observable or that cannot be detectable but gradient detectable and they provide a means to deal with some problems from the real world, for example in the thermic isolation problem it may be that the control is only required to vanish the temperature-gradient before crossing the wall.

Hence, with the same preoccupation, we introduce in this paper, a new concept that is gradient remediability of distributed parabolic systems. We recall that the notion of remediability consists in studying the existence of a convenient input operator (efficient actuators), ensuring the remediability of any disturbance acting in the considered systems. This problem is particularly motivated by pollution problems and so called space compensation or remediability problem. The notions of remediability and efficient actuators are introduced and studied first for discrete systems and there for continuous systems of a finite time horizon and for other situations (regional and asymptotic cases, internal or boundary actions of disturbances) [14-16].

This paper is organized as follows: In the second paragraph, we start by presenting the notations and some preliminary material. After, we recall the notions of exact and weak gradient controllability. In the third paragraph, we introduce and we characterize the notions 
of exact and weak gradient remadiability. We show how to find an input operator (actuators) with respect to the output (gradient observation) that ensures the gradient remediability of a disturbed parabolic system. By analogy with the relation between the remediability and the controllability examined in the finite time case, it is then natural to study, in the paragraph 4 , the relationship between the gradient remediability and the gradient controllability. We show that the gradient remediability is weaker and more supple than the gradient controllability of the parabolic systems, that is to say, if any parabolic systems are gradient controllable, then it is gradient remediable. The fifth paragraph recall the notion of gradient strategic actuators and we give a characterization of gradient remediability which shows that the gradient remediability of any system may depend on the structure of the actuators and sensors. Then we introduce and we characterize the notion of gradient efficient actuators. In paragraph 6, using an extension of Hilbert Uniqueness Method (H.U.M), we examine the problem of gradient remediability with minimum energy, and we give the optimal control that compensate an arbitrary disturbance. In the last paragraph, approximations and numerical results are presented.

\section{CONSIDERED SYSTEM}

Let $\Omega$ be an open and bounded regular subset of $\operatorname{IR}^{n}(n=1,2,3)$ with a smooth boundary $\partial \Omega$. For $T>0$, we denoted by $Q=\Omega \times] 0, T\left[, \quad \sum=\partial \Omega \times\right] 0, T[$. Consider a parabolic system defined by

$$
\begin{cases}\frac{\partial y}{\partial t}(x, t)=A y(x, t)+B u(t)+f(x, t) & Q \\ y(x, 0)=y^{0}(x) & \Omega \\ y(\xi, t)=0 & \Sigma\end{cases}
$$

Where $A$ is a second order linear differential operator with compact resolvent and which generates a strongly continuous semi-group $(S(t))_{t \geq 0}$ on the Hilbert space $L^{2}(\Omega) .\left(S^{*}(t)\right)_{t \geq 0}$ is considered for the adjoint semi-groupe of $(S(t))_{t \geq 0}$.

$B \in \mathscr{L}(U, X), u \in L^{2}(0, T ; U)$ where $U$ is a Hilbert space representing the control space and $X=H_{0}^{1}(\Omega)$ the state space. The disturbance term $f \in L^{2}(0, T ; X)$ is generally unknown. 
The system (1) admits a unique solution $y \in C\left(0, T ; H_{0}^{1}(\Omega)\right) \cap C^{1}\left(0, T ; L^{2}(\Omega)\right)$ [17] given by

$$
y(t)=S(t) y^{0}+\int_{0}^{t} S(t-s) B u(s) d s+\int_{0}^{t} S(t-s) f(s) d s
$$

and it is augmented by the output equation

$$
z(t)=C \nabla y(t)
$$

where $C \in \mathscr{L}\left(\left(L^{2}(\Omega)\right)^{n}, O\right), O$ is a Hilbert space (observation space). In the case of an observation on $[0, T]$ with $q$ sensors, we take generally $O=I R^{q}$. The operator $\nabla$ is defined by

$$
\begin{aligned}
\nabla: H_{0}^{1}(\Omega) & \rightarrow\left(L^{2}(\Omega)\right)^{n} \\
y & \rightarrow \nabla y=\left(\frac{\partial y}{\partial x_{1}}, \frac{\partial y}{\partial x_{2}}, \ldots, \frac{\partial y}{\partial x_{n}}\right)
\end{aligned}
$$

while $\nabla^{*}$ its adjoint operator.

\section{GRADIENT REMEDIABILITY}

The system (1) is disturbed by the force $f$ assumed unknown and excited by a control $u$ that will be chosen so as to compensate for the disturbance $f$. In the autonomous case, without disturbance $(f=0)$ and without $\operatorname{control}(u=0)$, this same system is written

$$
\begin{cases}\frac{\partial y}{\partial t}(x, t)=A y(x, t) & Q \\ y(x, 0)=y^{0}(x) & \Omega \\ y(\xi, t)=0 & \Sigma\end{cases}
$$

The system (3) admits a unique solution $y \in C\left(0, T ; H_{0}^{1}(\Omega)\right) \cap C^{1}\left(0, T ; L^{2}(\Omega)\right)[17]$ given by $y(t)=S(t) y^{0}$ then the observation on $[0, T]$ is normal and it is given by $z(t)=C \nabla S(t) y^{0}$. But if the control $u \neq 0$ and the disturbance $f \neq 0$, the observation noted $z_{u, f}$ is disturbed such that

$$
z_{u, f}(t)=C \nabla S(t) y^{0}+C \nabla \int_{0}^{t} S(t-s) B u(s) d s+C \nabla \int_{0}^{t} S(t-s) f(s) d s
$$

The problem consists to study the existence of an input operator $B$ (actuators), with respect to the output operator $C$ (sensors), ensuring at the time $T$, the gradient remediability of any 
disturbance on the system, that is:

For any $f \in L^{2}(0, T ; X)$, there exists $u \in L^{2}(0, T ; U)$ such that $z_{u, f}(T)=C \nabla S(T) y^{0}$ this is equivalent to $C \nabla H u+C \nabla F f=0$ where $H$ and $F$ are two operators defined by

$$
\begin{aligned}
H: L^{2}(0, T ; U) & \rightarrow X \\
u \rightarrow H u & =\int_{0}^{T} S(T-s) B u(s) d s \\
F: L^{2}(0, T ; X) & \rightarrow X \\
f \rightarrow F f & =\int_{0}^{T} S(T-s) f(s) d s
\end{aligned}
$$

This leads to the following definitions

\section{Definition 1:}

1) We say that the system (1) augmented by the output equation (2) is exactly gradient $f$ remediable on $[0, T]$, if there exists a control $u \in L^{2}(0, T ; U)$ such that

$$
C \nabla H u+C \nabla F f=0
$$

2) We say that the system (1) augmented by the output equation (2) is weakly gradient $f$ remediable on $[0, T]$, if for every $\varepsilon>0$, there exists a control $u \in L^{2}(0, T ; U)$ such that

$$
\|C \nabla H u+C \nabla F f\|_{O}<\varepsilon
$$

3) We say that the system (1) augmented by the output equation (2) is exactly (resp. weakly) gradient remediable on $[0, T]$, if for every $f \in L^{2}(0, T ; X)$ the system (1)-(2) is exactly (resp. weakly) gradient $f$-remediable.

\section{Proposition 1:}

Let us consider $f \in L^{2}(0, T ; X)$.

1) The system (1)-(2) is exactly gradient $f$-remediable on $[0, T]$ if and only if

$$
\operatorname{Im} C \nabla F f \in \operatorname{Im}(C \nabla H)
$$

2) The system (1)-(2) is weakly gradient $f$-remediable on $[0, T]$ if and only if

$$
\operatorname{Im} C \nabla F f \in \overline{\operatorname{Im}(C \nabla H)}
$$

\section{Proof:}

1) We assume that the system (1)-(2) is exactly gradient $f$-remediable on $[0, T]$, then there 
exists $u \in L^{2}(0, T ; U) \quad$ such that $C \nabla H u+C \nabla F f=0$, that is $C \nabla F f=-C \nabla H u=C \nabla H(-u)=C \nabla H u_{1} \quad$ with $\quad\left(u_{1}=-u\right) \quad u_{1} \in L^{2}(0, T ; U) \quad$ then $C \nabla F f \in \operatorname{Im} C \nabla H$.

Conversely, we assume that $C \nabla F f \in \operatorname{Im} C \nabla H$, then there exists $u \in L^{2}(0, T ; U)$ such that $C \nabla F f=C \nabla H u$ that is $C \nabla F f-C \nabla H u=0$ this gives $C \nabla F f+C \nabla H(-u)=0$. We put $u_{1}=-u \in L^{2}(0, T ; U)$ where the system (1)-(2) is exactly gradient $f$-remediable.

2) We assume that the system (1)-(2) is weakly gradient $f$-remediable on $[0, T]$, then $\forall \varepsilon>0, \exists u \in L^{2}(0, T ; U)$ such that $\|C \nabla F f+C \nabla H u\|_{O}<\varepsilon$ that is $\forall \varepsilon>0, \exists u \in L^{2}(0, T ; U)$ such that $\|C \nabla F f-C \nabla H(-u)\|_{O}<\varepsilon$. We put $u_{1}=-u \in L^{2}(0, T ; U)$, then $\forall \varepsilon>0, \exists u_{1} \in L^{2}(0, T ; U)$ such that $\left\|C \nabla F f-C \nabla H u_{1}\right\|_{O}<\varepsilon$, this gives $C \nabla F f \in \overline{\operatorname{Im}(C \nabla H)}$.

Conversely, we assume that $C \nabla F f \in \overline{\operatorname{Im}(C \nabla H)}$, then $\forall \varepsilon>0, \exists u_{1} \in L^{2}(0, T ; U)$ such that $\left\|C \nabla F f-C \nabla H u_{1}\right\|_{O}<\varepsilon$. We put $u_{1}=-u \in L^{2}(0, T ; U)$, then $\forall \varepsilon>0, \exists u \in L^{2}(0, T ; U)$ such that $\|C \nabla F f+C \nabla H u\|_{O}<\varepsilon$ where the system (1)-(2) is weakly gradient $f$-remediable.

\section{Proposition 2:}

1) The system (1)-(2) is exactly gradient remediable on $[0, T]$ if and only if

$$
\operatorname{Im} C \nabla F \subset \operatorname{Im} C \nabla H
$$

2) The system (1)-(2) is weakly gradient remediable on $[0, T]$ if and only if

$$
\operatorname{Im} C \nabla F \subset \overline{\operatorname{Im} C \nabla H}
$$

\section{Proof:}

1) We assume that the system (1)-(2) is exactly gradient remediable on $[0, T]$ then $\forall f \in L^{2}(0, T ; X)$ the system (1)-(2) is exactly gradient $f$ - remediable on $[0, T]$ and from the Proposition 1 we have, $\forall f \in L^{2}(0, T ; X): C \nabla F f \in \operatorname{Im} C \nabla H$, this gives $\operatorname{Im} C \nabla F \subset \operatorname{Im} C \nabla H$.

Conversely, we assume that $\operatorname{Im} C \nabla F \subset \operatorname{Im} C \nabla H$ and we show that the system (1)-(2) is exactly gradient remediable on $[0, T]$. Let $f \in L^{2}(0, T ; X)$ then $C \nabla F f \in \operatorname{Im} C \nabla F$ since 
$\operatorname{Im} C \nabla F \subset \operatorname{Im} C \nabla H$ we have $C \nabla F f \in \operatorname{Im} C \nabla H$ then there exists $u \in L^{2}(0, T ; U)$ such that $C \nabla F f=C \nabla H u$ that is to say $C \nabla F f-C \nabla H u=0$ this gives $C \nabla F f+C \nabla H(-u)=0$. We put $u_{1}=-u \in L^{2}(0, T ; U)$ where the system (1)-(2) is exactly gradient remediable.

2) We assume that the system (1)-(2) is weakly gradient remediable on $[0, T]$ then $\forall f \in L^{2}(0, T ; X)$ the system (1)-(2) is weakly gradient $f$-remediable on $[0, T]$ and from the Proposition.1 we have, $\forall f \in L^{2}(0, T ; X): C \nabla F f \in \overline{\operatorname{Im} C \nabla H}$, this gives $\operatorname{Im} C \nabla F \subset \overline{\operatorname{Im} C \nabla H}$.

Conversely, we assume that $\operatorname{Im} C \nabla F \subset \overline{\operatorname{Im} C \nabla H}$ and we show that the system (1)-(2) is weakly gradient remediable on $[0, T]$. Let $f \in L^{2}(0, T ; X)$ then $C \nabla F f \in \operatorname{Im} C \nabla F$. Since $\operatorname{Im} C \nabla F \subset \overline{\operatorname{Im} C \nabla H}$ then $C \nabla F f \in \overline{\operatorname{Im}(C \nabla H)}$ this leads to $\forall \varepsilon>0, \exists u \in L^{2}(0, T ; U)$ such that $\|C \nabla F f-C \nabla H u\|<\varepsilon$ by putting $u_{1}=-u \in \quad L^{2}(0, T ; U)$ this gives $\forall \varepsilon>0, \exists u_{1} \in L^{2}(0, T ; U)$ such that $\left\|C \nabla F f+C \nabla H u_{1}\right\|_{O}<\varepsilon$ where the system (1)-(2) is weakly gradient remediable on $[0, T]$.

\section{GRADIENT REMEDIABILITY AND GRADIENT CONTROLLABILITY}

By analogy with the relation between the remediability and the controllability examined in the finite time case, it is then natural to study, in this paragraph, the relationship between the gradient remediability and the gradient controllability. Firstly, we recall the definitions of exact and weak controllability.

\section{Definition 2:}

1) The system (1) is said to be exactly gradient controllable on $[0, T]$ if $\forall y^{d} \in\left(L^{2}(\Omega)\right)^{n}$ there exist a control $u \in L^{2}(0, T ; U)$ such that $\nabla y(T)=y^{d}$.

2) The system (1) is said weakly gradient controllable on $[0, T]$ if $\forall y^{d} \in\left(L^{2}(\Omega)\right)^{n}, \forall \varepsilon>0$ there exist a control $u \in L^{2}(0, T ; U)$ such that $\left\|\nabla y(T)-y^{d}\right\|_{\left(L^{2}(\Omega)\right)^{n}}<\varepsilon$. 


\section{Proposition 3:}

1) If the system (1)-(2) is exactly gradient controllable on $[0, T]$, then it is exactly gradient remediable on $[0, T]$.

2) If the system (1)-(2) is weakly gradient controllable on $[0, T]$, then it is weakly gradient remediable on $[0, T]$.

\section{Proof:}

1) We assume that the system (1)-(2) is exactly gradient controllable and let $y^{d}=S(T) y^{0}$ then there exists $u \in L^{2}(0, T ; U)$ such that $\nabla y(T)=\nabla S(T) y^{0}$ that is to say $\nabla S(T) y^{0}+\nabla H u+\nabla F f=\nabla S(T) y^{0} \quad$ this $\quad$ leads $\quad$ to $\quad \nabla H u+\nabla F f=0$ then let $C \nabla H u+C \nabla F f=0$ and then the system (1) - (2) is exactly gradient remediable.

2) We assume that the system (1)-(2) is weakly gradient controllable and let $y^{d}=\nabla S(T) y^{0}$ then $\forall \varepsilon>0, \quad \exists u \in L^{2}(0, T ; U) \quad$ such that $\left\|\nabla y(T)-\nabla S(T) y^{0}\right\|_{\left(L^{2}(\Omega)\right)^{n}}<\varepsilon \quad$ that is $\|\nabla H u+\nabla F f\|_{\left(L^{2}(\Omega)\right)^{n}}<\varepsilon$. Since the operator $C$ is continue, consequently we have $\|C \nabla H u+C \nabla F f\|_{O} \leq k\|\nabla H u+\nabla F f\|_{\left(L^{2}(\Omega)\right)^{n}}$ with $k>0$ where the system (1)-(2) is weakly gradient remediable.

\section{GRADIENT REMEDIABILITY, SENSORS AND ACTUATORS}

We suppose that the system (1) is excited by $p$ zone actuators $\left(\Omega_{i}, g_{i}\right)_{1 \leq i \leq p}, g_{i} \in L^{2}\left(\Omega_{i}\right)$, $\Omega_{i}=\operatorname{supp}\left(g_{i}\right) \subset \Omega$ in this case the control space is $U=I R^{p}$ and the operator:

$$
\begin{aligned}
& B: I^{p} \rightarrow X \\
& u(t)=\left(u_{1}(t), u_{2}(t), \ldots, u_{p}(t)\right) \mapsto B u=\sum_{i=1}^{p} \chi_{\Omega_{i}}(x) g_{i}(x) u_{i}(t)
\end{aligned}
$$

Its adjoint is given by

$$
B^{*} z=\left(\left\langle g_{1}, z\right\rangle_{\Omega_{1}},\left\langle g_{2}, z\right\rangle_{\Omega_{2}}, \ldots,\left\langle g_{p}, z\right\rangle_{\Omega_{p}}\right)^{T} \in I R^{p}
$$


Also suppose that the output of the system (2) is given by $q$ sensors $\left(D_{i}, h_{i}\right)_{1 \leq i \leq q}, h_{i} \in L^{2}\left(D_{i}\right)$, being the spatial distribution, $D_{i}=\operatorname{supp} h_{i} \subset \Omega$, for $i=1, \ldots, q$ and $D_{i} \cap D_{j}=\phi$ for $i \neq j$, then the operator $C$ is defined by:

$$
\begin{aligned}
& C:\left(L^{2}(\Omega)\right)^{n} \rightarrow I R^{q} \\
& C y(t)=\left(\sum_{i=1}^{n}\left\langle h_{1}, y_{i}(t)\right\rangle_{D_{1}}, \sum_{i=1}^{n}\left\langle h_{2}, y_{i}(t)\right\rangle_{D_{2}}, \ldots, \sum_{i=1}^{n}\left\langle h_{q}, y_{i}(t)\right\rangle_{D_{q}}\right)^{T}
\end{aligned}
$$

its adjoint is given by $C^{*}$ with for $\theta=\left(\theta_{1}, \theta_{2}, \ldots, \theta_{q}\right)^{T} \in I R^{q}$

$$
C^{*} \theta=\left(\sum_{i=1}^{q} \chi_{D_{i}}(x) \theta_{i} h_{i}(x), \sum_{i=1}^{q} \chi_{D_{i}}(x) \theta_{i} h_{i}(x), \ldots, \sum_{i=1}^{q} \chi_{D_{i}}(x) \theta_{i} h_{i}(x)\right)
$$

\section{Lemma 1 [18]:}

Let $V, W$ and $Z$ be reflexive Banach spaces, $P \in \mathscr{L}(V, Z)$ and $Q \in \mathscr{L}(W, Z)$. Then the following properties are equivalent:

$$
\begin{aligned}
& \text { i. } \operatorname{Im} P \subset \operatorname{Im} Q \\
& \text { ii. } \exists \gamma>0 \text { such that }\left\|P^{*} z^{*}\right\|_{V^{\prime}} \leq \gamma\left\|Q^{*} z^{*}\right\|_{W^{\prime}}, \quad \forall z^{*} \in Z^{\prime}
\end{aligned}
$$

We have the following characterizations:

\section{Proposition 4:}

1) The system (1)-(2) is exactly gradient remediable on $[0, T]$ if and only if there exists $\gamma>0$ such that for every $\theta \in I R^{q}$, we have

$$
\left\|S^{*}(T-.) \nabla^{*} C^{*} \theta\right\|_{L^{2}\left(0, T ; X^{\prime}\right)} \leq \gamma\left\|B^{*} S^{*}(T-.) \nabla^{*} C^{*} \theta\right\|_{L^{2}\left(0, T ; I R^{p}\right)}
$$

2) The system (1) - (2) is weakly gradient remediable on $[0, T]$ if and only if

$$
\operatorname{ker}\left(B^{*} F^{*} \nabla^{*} C^{*}\right)=\operatorname{ker}\left(F^{*} \nabla^{*} C^{*}\right)
$$

\section{Proof:}

1) It follows from the fact that $F^{*} \nabla^{*} C^{*}=S^{*}(T-.) \nabla^{*} C^{*}$ and that $H^{*} \nabla^{*} C^{*}=B^{*} S^{*}(T-.) \nabla^{*} C^{*}$ and since the Proposition 2, we put $P=C \nabla F$ and $Q=C \nabla H$ and using the Lemma 1.

2) We assume that the system (1)-(2) is weakly gradient remediable on $[0, T]$ and we show that $\operatorname{ker}\left(B^{*} F^{*} \nabla^{*} C^{*}\right)=\operatorname{ker}\left(F^{*} \nabla^{*} C^{*}\right)$. Let $\theta \in I R^{q}$ such that $B^{*} F^{*} \nabla^{*} C^{*} \theta=0$, and we have 


$$
\left\{\begin{array}{c}
F^{*}=S^{*}(T-.) \\
H^{*}=B^{*} S^{*}(T-.)
\end{array}\right.
$$

then, $\quad B^{*} F^{*} \nabla^{*} C^{*} \theta=0 \Rightarrow H^{*} \nabla^{*} C^{*} \theta=0$ this gives $\theta \in \operatorname{ker}\left(H^{*} \nabla^{*} C^{*}\right)$ and we have $\overline{\operatorname{Im}(C \nabla H)}=\left[\operatorname{ker}\left(H^{*} \nabla^{*} C^{*}\right)\right]^{\perp}$. Since the hypothesis and the Proposition 2, we have $\operatorname{Im} C \nabla F \subset \overline{\operatorname{Im} C \nabla H} \quad$ then $\quad \operatorname{Im} C \nabla F \subset\left[\operatorname{ker}\left(H^{*} \nabla^{*} C^{*}\right)\right]^{\perp} \quad \Rightarrow \forall f \in L^{2}(0, T ; X): C \nabla F f \in$ $\left[\operatorname{ker}\left(H^{*} \nabla^{*} C^{*}\right)\right]^{\perp} \quad \Rightarrow\langle C \nabla F f, \theta\rangle=0 \quad$ because $\quad \theta \in \operatorname{ker}\left(H^{*} \nabla^{*} C^{*}\right), \quad$ this gives $\theta \in[\operatorname{Im} C \nabla F]^{\perp}=\operatorname{ker}\left(F^{*} \nabla^{*} C^{*}\right)$, where the result.

Conversely, assume that $\operatorname{ker}\left(B^{*} F^{*} \nabla^{*} C^{*}\right)=\operatorname{ker}\left(F^{*} \nabla^{*} C^{*}\right)$ and we show that $\operatorname{Im} C \nabla F \subset \overline{\operatorname{ImC} C H}$. Let $f \in L^{2}(0, T ; X)$ such that $f \in \operatorname{ImC} C F$, we have $\overline{\operatorname{ImC} C H}=\left[\operatorname{ker}\left(H^{*} \nabla^{*} C^{*}\right)\right]^{\perp}$. For every $\theta \in I R^{q}$ such that $H^{*} \nabla^{*} C^{*} \theta=0$, that is $B^{*} F^{*} \nabla^{*} C^{*} \theta=0$ we have $F^{*} \nabla^{*} C^{*} \theta=0$ because $\operatorname{ker}\left(B^{*} F^{*} \nabla^{*} C^{*}\right)=\operatorname{ker}\left(F^{*} \nabla^{*} C^{*}\right)$ then $\langle C \nabla F f, \theta\rangle=0$, where the result.

\section{Corollary 1:}

The system (1)-(2) is exactly gradient remediable on $[0, T]$ if and only if $\exists \gamma>0$ such that $\forall \theta \in I R^{q}$, we have

$$
\int_{0}^{T}\left\|S^{*}(T-s) \nabla^{*} C^{*} \theta\right\|_{X^{\prime}}^{2} d s \leq \gamma \sum_{i=1}^{p} \int_{0}^{T}\left\langle g_{i}, S^{*}(T-s) \nabla^{*} C^{*} \theta\right\rangle_{L^{2}\left(\Omega_{i}\right)}^{2} d s
$$

\section{Proof:}

Since the Proposition 4, the system (1)-(2) is exactly gradient remediable on $[0, T]$ if and only if there exists $\gamma>0$ such that for every $\theta \in I R^{q}$, we have

$$
\left\|S^{*}(T-.) \nabla^{*} C^{*} \theta\right\|_{L^{2}\left(0, T ; X^{\prime}\right)}^{2} \leq \gamma\left\|B^{*} S^{*}(T-.) \nabla^{*} C^{*} \theta\right\|_{L^{2}\left(0, T ; I R^{p}\right)}^{2}
$$

by using (4) the formula of the operator $B^{*}$, we have

$$
\int_{0}^{T}\left\|S^{*}(T-s) \nabla^{*} C^{*} \theta\right\|_{X^{\prime}}^{2} d s \leq \gamma \sum_{i=1}^{p} \int_{0}^{T}\left\langle g_{i}, S^{*}(T-s) \nabla^{*} C^{*} \theta\right\rangle^{2} d s
$$

where the result.

In the following, without loss of generality we consider, the system (1) with a dynamics $A$ of the form 


$$
A y=\sum_{m \geq 1} \lambda_{m} \sum_{j=1}^{r_{m}}\left\langle y, w_{m j}\right\rangle_{L^{2}(\Omega)} w_{m j}, \forall y \in D(A)=H^{2}(\Omega) \cap H_{0}^{1}(\Omega)
$$

where $\left(w_{m j}\right)_{\substack{1 \leq j \leq r_{m} \\ m \geq 1}}$ is an orthogonal basis in $H_{0}^{1}(\Omega)$ of eigenvectors of $A$ orthonormal in $L^{2}(\Omega)$, associated to eigenvalues $\lambda_{m}<0$ with a multiplicity $r_{m}$. Then, the operator $A$ generates on the Hilbert space $L^{2}(\Omega)$ a strongly continuous semi-group $(S(t))_{t \geq 0}$ given by $[1,19]$ :

$$
S(t) y=\sum_{m \geq 1} e^{\lambda_{m} t} \sum_{j=1}^{r_{m}}\left\langle y, w_{m j}\right\rangle_{L^{2}(\Omega)} w_{m j}
$$

\section{Corollary 2:}

The system (1)-(2) is exactly gradient remediable on $[0, T]$ if and only if $\exists \gamma>0$ such that $\forall \theta \in I R^{q}$, we have

$$
\sum_{m \geq 1} \frac{1}{2 \lambda_{m}}\left(e^{2 \lambda_{m} T}-1\right) \sum_{j=1}^{r_{m}}\left\langle C^{*} \theta, \nabla w_{m j}\right\rangle_{\left(L^{2}(\Omega)\right)^{n}}^{2} \leq \gamma \sum_{i=1}^{p} \int_{0}^{T}\left(\sum_{m \geq 1} e^{\lambda_{m}(T-s)} \sum_{j=1}^{r_{m}}\left\langle C^{*} \theta, \nabla w_{m j}\right\rangle\left\langle g_{i}, w_{m j}\right\rangle\right)^{2} d s
$$

\section{Proof:}

Since the Corollary 1, the system (1)-(2) is exactly gradient remediable on $[0, T]$ if and only if there exists $\gamma>0$ such that for every $\theta \in I R^{q}$, we have

$$
\int_{0}^{T}\left\|S^{*}(T-s) \nabla^{*} C^{*} \theta\right\|_{X^{\prime}}^{2} d s \leq \gamma \sum_{i=1}^{p} \int_{0}^{T}\left\langle g_{i}, S^{*}(T-s) \nabla^{*} C^{*} \theta\right\rangle_{\Omega_{i}}^{2} d s
$$

By using (6) the formula of the operator $S$ and since it is auto-adjoint, we obtain

$$
\begin{aligned}
& \int_{0}^{T}\left\|S^{*}(T-S) \nabla^{*} C^{*} \theta\right\|_{L^{2}(\Omega)}^{2} d s=\int_{0}^{T} \sum_{m \geq 1} e^{2 \lambda_{m}(T-s)} \sum_{j=1}^{r_{m}}\left\langle\nabla^{*} C^{*} \theta, w_{m j}\right\rangle^{2} d s \\
& =\sum_{m \geq 1} \frac{1}{2 \lambda_{m}}\left(e^{2 \lambda_{m} T}-1\right) \sum_{j=1}^{r_{m}}\left\langle C^{*} \theta, \nabla w_{m j}\right\rangle^{2}
\end{aligned}
$$

and

$$
\sum_{i=1}^{p} \int_{0}^{T}\left\langle g_{i}, S^{*}(T-s) \nabla^{*} C^{*} \theta\right\rangle_{L^{2}\left(\Omega_{i}\right)}^{2} d s=\sum_{i=1}^{p} \int_{0}^{T}\left(\sum_{m \geq 1} e^{\lambda_{m}(T-s)} \sum_{j=1}^{r_{m}}\left\langle\nabla^{*} C^{*} \theta, w_{m j}\right\rangle\left\langle g_{i}, w_{m j}\right\rangle\right)^{2} d s
$$

where the result.

By using (5) the formula of the operator $C^{*}$, we have the following corollary: 


\section{Corollary 3:}

The system (1)-(2) is exactly gradient remediable on $[0, T]$ if and only if $\exists \gamma>0$ such that $\forall \theta \in I R^{q}$, we have

$$
\begin{aligned}
& \sum_{m \geq 1} \frac{1}{2 \lambda_{m}}\left(e^{2 \lambda_{m} T}-1\right) \sum_{j=1}^{r_{m}} \sum_{k=1}^{n} \sum_{l=1}^{q}\left\langle\theta_{l} h_{l}, \frac{\partial w_{m j}}{\partial x_{k}}\right\rangle_{L^{2}\left(D_{l}\right)}^{2} \\
& \leq \gamma \sum_{i=1}^{p} \int_{0}^{T}\left(\sum_{m \geq 1} e^{\lambda_{m}(T-s)} \sum_{j=1}^{r_{m}}\left\langle g_{i}, w_{m j}\right\rangle_{L^{2}\left(\Omega_{i}\right)} \sum_{k=1}^{n} \sum_{l=1}^{q} \theta_{l}\left\langle h_{l}, \frac{\partial w_{m j}}{\partial x_{k}}\right\rangle\right)^{2} d s
\end{aligned}
$$

\section{Proof:}

Since the Corollary 2, the system (1)-(2) is exactly gradient remediable on $[0, T]$ if and only if there exists $\gamma>0$ such that for every $\theta \in I R^{q}$, we have

$$
\sum_{m \geq 1} \frac{1}{2 \lambda_{m}}\left(e^{2 \lambda_{m} T}-1\right) \sum_{j=1}^{r_{m}}\left\langle C^{*} \theta, \nabla w_{m j}\right\rangle_{\left(L^{2}(\Omega)\right)^{n}}^{2} \leq \gamma \sum_{i=1}^{p} \int_{0}^{T}\left(\sum_{m \geq 1} e^{\lambda_{m}(T-s)} \sum_{j=1}^{r_{m}}\left\langle C^{*} \theta, \nabla w_{m j}\right\rangle\left\langle g_{i}, w_{m j}\right\rangle\right)^{2} d s
$$

and by using (5) the formula of the operator $C^{*}$, we have

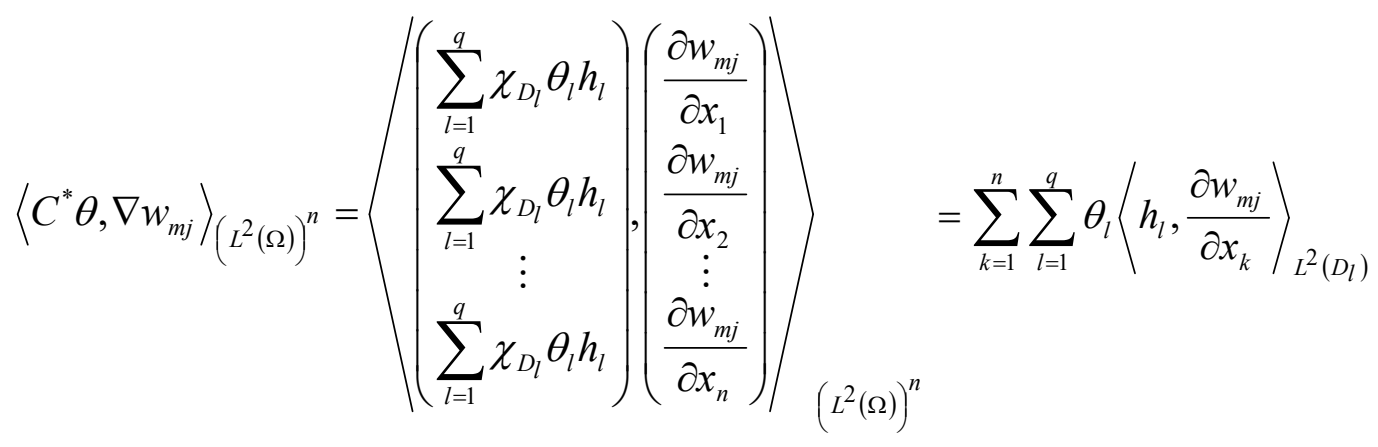

where the result.

This characterization shows that the remediability of a system may depend on the structure of the actuators and sensors.

By analogy with the concept of gradient strategic actuator, we introduce the notion of gradient efficient actuator, as follows:

\section{Definition 3:}

The actuators $\left(\Omega_{i}, g_{i}\right)_{1 \leq i \leq p}, g_{i} \in L^{2}\left(\Omega_{i}\right)$ are said to be gradient efficient if the system (1)-(2) so excited is weakly gradient remediable.

The actuators gradient efficient define actions with the structure (spatial distribution, location and number) can compensate the effect of disturbance distributed on the system. We have then 
the following characterization of the gradient efficient actuators.

For $m \geq 1$, let $M_{m}$ be the matrix of order $\left(p \times r_{m}\right)$ defined by $M_{m}=\left(\left\langle g_{i}, w_{m j}\right\rangle\right)_{i j}, 1 \leq i \leq p$ and $1 \leq j \leq r_{m}$ and let $G_{m}$ be the matrix of order $\left(q \times r_{m}\right)$ defined by $G_{m}=\left(\sum_{k=1}^{n}\left\langle h_{i}, \frac{\partial w_{m j}}{\partial x_{k}}\right\rangle\right)_{i j}, 1 \leq i \leq q \quad$ and $\quad 1 \leq j \leq r_{m}$.

\section{Proposition 5:}

The actuators $\left(\Omega_{i}, g_{i}\right)_{1 \leq i \leq p}, g_{i} \in L^{2}\left(\Omega_{i}\right)$ are gradient efficient if and only if $\operatorname{ker}\left(\nabla^{*} C^{*}\right)=\bigcap_{m \geq 1} \operatorname{ker}\left(M_{m} f_{m}\right)$. Where, for $\theta \in I R^{q}$ and $m \geq 1$,

$$
f_{m}(\theta)=\left(\left\langle\nabla^{*} C^{*} \theta, w_{m 1}\right\rangle,\left\langle\nabla^{*} C^{*} \theta, w_{m 2}\right\rangle, \ldots,\left\langle\nabla^{*} C^{*} \theta, w_{m r_{m}}\right\rangle\right)^{T} \in I R^{r_{m}}
$$

\section{Proof:}

We assume that the actuators $\left(\Omega_{i}, g_{i}\right)_{1 \leq i \leq p}, g_{i} \in L^{2}\left(\Omega_{i}\right)$ are gradient efficient and we show that $\operatorname{ker}\left(\nabla^{*} C^{*}\right)=\bigcap_{m \geq 1} \operatorname{ker}\left(M_{m} f_{m}\right)$. Since the Proposition 4, the system (1)-(2) is weakly gradient remediable on $[0, T]$ if and only if $\operatorname{ker}\left(B^{*} F^{*} \nabla^{*} C^{*}\right)=\operatorname{ker}\left(F^{*} \nabla^{*} C^{*}\right)$. Let $\theta \in I R^{q}$, we have

$$
B^{*} F^{*} \nabla^{*} C^{*} \theta=B^{*} S^{*}(T-.) \nabla^{*} C^{*} \theta=\left(\begin{array}{c}
\sum_{m \geq 1} e^{\lambda_{m}(T-.)} \sum_{j=1}^{r_{m}}\left\langle\nabla^{*} C^{*} \theta, w_{m j}\right\rangle_{L^{2}(\Omega)}\left\langle g_{1}, w_{m j}\right\rangle_{L^{2}\left(\Omega_{1}\right)} \\
\sum_{m \geq 1} e^{\lambda_{m}(T-.)} \sum_{j=1}^{r_{m}}\left\langle\nabla^{*} C^{*} \theta, w_{m j}\right\rangle_{L^{2}(\Omega)}\left\langle g_{2}, w_{m j}\right\rangle_{L^{2}\left(\Omega_{2}\right)} \\
\vdots \\
\sum_{m \geq 1} e^{\lambda_{m}(T-.)} \sum_{j=1}^{r_{m}}\left\langle\nabla^{*} C^{*} \theta, w_{m j}\right\rangle_{L^{2}(\Omega)}\left\langle g_{P}, w_{m j}\right\rangle_{L^{2}\left(\Omega_{p}\right)}
\end{array}\right)
$$

and we have $\forall m \geq 1$,

$$
M_{m} f_{m}(\theta)=\left(\begin{array}{c}
\sum_{j=1}^{r_{m}}\left\langle\nabla^{*} C^{*} \theta ; w_{m j}\right\rangle_{L^{2}(\Omega)}\left\langle g_{1}, w_{m j}\right\rangle_{L^{2}\left(\Omega_{1}\right)} \\
\sum_{j=1}^{r_{m}}\left\langle\nabla^{*} C^{*} \theta ; w_{m j}\right\rangle_{L^{2}(\Omega)}\left\langle g_{2}, w_{m j}\right\rangle_{L^{2}\left(\Omega_{2}\right)} \\
\vdots \\
\sum_{j=1}^{r_{m}}\left\langle\nabla^{*} C^{*} \theta ; w_{m j}\right\rangle_{L^{2}(\Omega)}\left\langle g_{P}, w_{m j}\right\rangle_{L^{2}\left(\Omega_{p}\right)}
\end{array}\right)
$$

If we assume that $\theta \in \bigcap_{m \geq 1} \operatorname{ker}\left(M_{m} f_{m}\right)$, then 


$$
\begin{aligned}
& \theta \in \operatorname{ker}\left(M_{m} f_{m}\right), \forall m \geq 1 \\
& \Rightarrow \sum_{j=1}^{r_{m}}\left\langle\nabla^{*} C^{*} \theta ; w_{m j}\right\rangle_{L^{2}(\Omega)}\left\langle g_{i}, w_{m j}\right\rangle=0, \forall i \in\{1,2, \ldots, p\}, \forall m \geq 1 \\
& \Rightarrow \sum_{m \geq 1} e^{\lambda_{m}(T-.)} \sum_{j=1}^{r_{m}}\left\langle\nabla^{*} C^{*} \theta, w_{m j}\right\rangle\left\langle g_{i}, w_{m j}\right\rangle=0, \forall i \in\{1,2, \ldots, p\}, \forall m \geq 1 \\
& \Rightarrow B^{*} F^{*} \nabla^{*} C^{*} \theta=0 \Rightarrow \theta \in \operatorname{ker}\left(B^{*} F^{*} \nabla^{*} C^{*}\right) \text { where } \bigcap_{m \geq 1} \operatorname{ker}\left(M_{m} f_{m}\right) \subset \operatorname{ker}\left(B^{*} F^{*} \nabla^{*} C^{*}\right) \text { that is } \\
& \bigcap_{m \geq 1} \operatorname{ker}\left(M_{m} f_{m}\right)=\operatorname{ker}\left(B^{*} F^{*} \nabla^{*} C^{*}\right) .
\end{aligned}
$$

On the other hand, we have for every $\theta \in I R^{q}$, $F^{*} \nabla^{*} C^{*} \theta=S^{*}(T-.) \nabla^{*} C^{*}=\sum_{m \geq 1} e^{\lambda_{m}(T-.)} \sum_{j=1}^{r_{m}}\left\langle\nabla^{*} C^{*} \theta, w_{m j}\right\rangle w_{m j}$ We assume that $\theta \in \operatorname{ker}\left(F^{*} \nabla^{*} C^{*}\right)$, then $F^{*} \nabla^{*} C^{*} \theta=0$

$$
F^{*} \nabla^{*} C^{*} \theta=S^{*}(T-.) \nabla^{*} C^{*}=\sum_{m \geq 1} e^{\lambda_{m}(T-.)} \sum_{j=1}^{r_{m}}\left\langle\nabla^{*} C^{*} \theta, w_{m j}\right\rangle w_{m j}=0
$$$$
\Rightarrow \sum_{m \geq 1} \sum_{j=1}^{r_{m}}\left\langle\nabla^{*} C^{*} \theta, w_{m j}\right\rangle w_{m j}=0 \Rightarrow \nabla^{*} C^{*} \theta=0 \Leftrightarrow \theta \in \operatorname{ker}\left(\nabla^{*} C^{*}\right) \text {. Then, }
$$

$\operatorname{ker}\left(F^{*} \nabla^{*} C^{*}\right) \subset \operatorname{ker}\left(\nabla^{*} C^{*}\right)$. If we assume that $\theta \in \operatorname{ker}\left(\nabla^{*} C^{*}\right)$, then $\nabla^{*} C^{*} \theta=0$ that is

$F^{*} \nabla^{*} C^{*} \theta=\sum_{m \geq 1} e^{\lambda_{m}(T-.)} \sum_{j=1}^{r_{m}}\left\langle\nabla^{*} C^{*} \theta, w_{m j}\right\rangle w_{m j}=0 \Rightarrow \theta \in \operatorname{ker}\left(F^{*} \nabla^{*} C^{*}\right)$, then

$$
\operatorname{ker}\left(\nabla^{*} C^{*}\right) \subset \operatorname{ker}\left(F^{*} \nabla^{*} C^{*}\right) \text { that is } \operatorname{ker}\left(\nabla^{*} C^{*}\right)=\operatorname{ker}\left(F^{*} \nabla^{*} C^{*}\right) \text {. }
$$

Where the result.

\section{Corollary 4:}

If there exists $m_{0} \geq 1$ such that

$$
\operatorname{rank} G_{m_{0}}^{T}=q
$$

then the actuators $\left(\Omega_{i}, g_{i}\right)_{1 \leq i \leq p}, g_{i} \in L^{2}\left(\Omega_{i}\right)$ are efficient if and only if $\bigcap_{m \geq 1} \operatorname{ker}\left(M_{m} G_{m}^{T}\right)=\{0\}$.

\section{Proof:}

Let $\theta \in I R^{q}$, then $\theta \in \bigcap_{m \geq 1} \operatorname{ker}\left(M_{m} G_{m}^{T}\right) \Leftrightarrow\left(M_{m} G_{m}^{T}\right) \theta=0, \quad \forall m \geq 1$

$$
\Leftrightarrow \sum_{l=1}^{q} \sum_{j=1}^{r_{m}}\left\langle g_{i}, w_{m_{j}}\right\rangle\left\langle h_{l}, \sum_{k=1}^{n} \frac{\partial w_{m_{j}}}{\partial x_{k}}\right\rangle \theta_{l}=0, \forall m \geq 1, \forall i=1, \ldots, p
$$




$$
\begin{aligned}
& \Leftrightarrow \sum_{j=1}^{r_{m}}\left\langle g_{i}, w_{m j}\right\rangle\left\langle\nabla^{*} C^{*}, w_{m j}\right\rangle_{L^{2}(\Omega)}=0, \forall m \geq 1, \forall i=1, \ldots, p \\
& \Leftrightarrow\left(M_{m} f_{m}\right) \theta=0, \forall m \geq 1 \Leftrightarrow \theta \in \bigcap_{m \geq 1} \operatorname{ker}\left(M_{m} f_{m}\right) \text { this gives } \bigcap_{m \geq 1} \operatorname{ker}\left(M_{m} G_{m}^{T}\right)=\bigcap_{m \geq 1} \operatorname{ker}\left(M_{m} f_{m}\right) .
\end{aligned}
$$

On the other hand, $\theta \in \operatorname{ker}\left(\nabla^{*} C^{*}\right) \Leftrightarrow \nabla^{*} C^{*} \theta=0$, then for $m_{0}$ that appears in the hypothesis and by using (5) the formula of the operator $C^{*}$, we obtain

$$
\left\langle\nabla^{*} C^{*} \theta, w_{m_{0} j}\right\rangle=\sum_{l=1}^{q} \theta_{l}\left\langle h_{l}, \sum_{k=1}^{n} \frac{\partial w_{m_{0} j}}{\partial x_{k}}\right\rangle_{L^{2}\left(D_{l}\right)}=0, \forall j=1, \ldots, r_{m_{0}} \Rightarrow G_{m_{0}}^{T} \theta=0 \Leftrightarrow \theta \in \operatorname{ker} G_{m_{0}}^{T} \quad \text { and }
$$

since $\operatorname{rank} G_{m_{0}}^{T}=q$, then $\operatorname{ker} G_{m_{0}}^{T}=\{0\}$ this gives $\theta=0$. That is $\operatorname{ker}\left(\nabla^{*} C^{*}\right)=\{0\}$

Finally, the proof follows directly from the Proposition 5.

\section{Corollary 5:}

If there exists $m_{0} \geq 1$ such that $\operatorname{rank}\left(G_{m_{0}}^{T}\right)=q$ and if

$$
\operatorname{rank}\left(M_{m_{0}} G_{m_{0}}^{T}\right)=q
$$

Or

$$
\operatorname{rank}\left(M_{m_{0}}\right)=r_{m_{0}}
$$

then the actuators $\left(\Omega_{i}, g_{i}\right)_{1 \leq i \leq p}, g_{i} \in L^{2}\left(\Omega_{i}\right)$ are gradient efficient.

\section{Proof:}

Assume that there exists $m_{0} \geq 1$ such that $\operatorname{rank}\left(M_{n_{0}} G_{n_{0}}^{T}\right)=q$. The matrix $\left(M_{m_{0}} G_{m_{0}}^{T}\right)$ is of order $p \times q$. From the theorem of rank to matrices [20], we have $\operatorname{rank}\left(M_{m_{0}} G_{m_{0}}^{T}\right)+\operatorname{dim}\left(\operatorname{ker}\left(M_{m_{0}} G_{m_{0}}^{T}\right)\right)=q$, and then $\operatorname{dim}\left(\operatorname{ker}\left(M_{m_{0}} G_{m_{0}}^{T}\right)\right)=0$ witch is

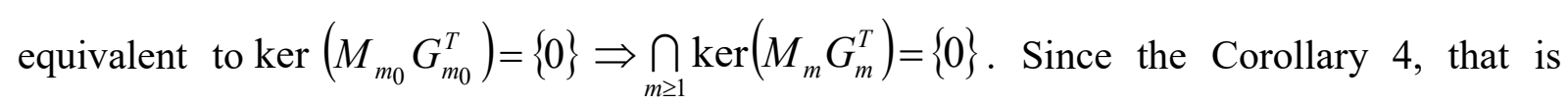
equivalent to the gradient efficient of the actuators $\left(\Omega_{i}, g_{i}\right)_{1 \leq i \leq p}, g_{i} \in L^{2}\left(\Omega_{i}\right)$.

Now, we suppose that $\operatorname{rank}\left(G_{m_{0}}^{T}\right)=q$ and $\operatorname{rank}\left(M_{m_{0}}\right)=r_{m_{0}}$. The matrix $\left(G_{m_{0}}^{T}\right)$ is of order $r_{m_{0}} \times q$. By using the theorem of rank to matrices [20], we have $\operatorname{rank}\left(G_{m_{0}}^{T}\right)+\operatorname{dim}\left(\operatorname{ker}\left(G_{m_{0}}^{T}\right)\right)=q$ then, $\operatorname{dim}\left(\operatorname{ker}\left(G_{m_{0}}^{T}\right)\right)=0$. That is equivalent to

$$
\operatorname{ker}\left(G_{m_{0}}^{T}\right)=\{0\}
$$

The same, the matrix $\left(M_{m_{0}}\right)$ is of order $p \times r_{m_{0}}$. By using the theorem of rank for matrices 
[19], we have rang $\left(M_{m_{0}}\right)+\operatorname{dim}\left(\operatorname{ker}\left(M_{m_{0}}\right)\right)=r_{m_{0}}$. And from (9), we obtain $\operatorname{dim}\left(\operatorname{ker}\left(M_{m_{0}}\right)\right)=0 \quad$ which is equivalent to

$$
\operatorname{ker}\left(M_{m_{0}}\right)=\{0\}
$$

On the other hand, let $\theta \in \operatorname{ker}\left(M_{m_{0}} G_{m_{0}}^{T}\right)$, then $\left(M_{m_{0}} G_{m_{0}}^{T}\right) \theta=0$ which gives $M_{m_{0}}\left(G_{m_{0}}^{T} \theta\right)=0$. From (11), we obtain $G_{m_{0}}^{T} \theta=0$ and from (10), we obtain $\theta=0$ then $\operatorname{ker}\left(M_{m_{0}} G_{m_{0}}^{T}\right)=\{0\}$ and then, $\bigcap_{m \geq 1} \operatorname{ker}\left(M_{m} G_{m}^{T}\right)=\{0\}$ which is equivalent, from the Corollary 4 , to the gradient efficient of the actuators $\left(\Omega_{i}, g_{i}\right)_{1 \leq i \leq p}, g_{i} \in L^{2}\left(\Omega_{i}\right)$.

\section{Remark 1:}

1) The condition (8) $\Rightarrow q \leq p$.

2) The condition $q \leq p$ is not necessary for actuators to be gradient efficient. Indeed, in the case of a single actuator $\left(\Omega_{1}, g\right)$ and of $q$ sensors $\left(D_{i}, h_{i}\right)_{1 \leq i \leq q}$, with $q>1$, $M_{m}=\left(\left\langle g, w_{m j}\right\rangle\right)_{1 \leq j \leq r_{m}}$ is of order $\left(1 \times r_{m}\right)$ and $G_{m}^{T}=\left(\sum_{k=1}^{n}\left\langle h_{i}, \frac{\partial w_{m j}}{\partial x_{k}}\right\rangle\right)_{\substack{1 \leq j \leq r_{m} \\ 1 \leq i \leq q}}$ is of order $\left(r_{m} \times q\right) \quad$ consequently $M_{m} G_{m}^{T}=\left(\sum_{j=1}^{r_{m}} \sum_{k=1}^{n}\left\langle g, w_{m j}\right\rangle\left\langle h_{i}, \frac{\partial w_{m j}}{\partial x_{k}}\right\rangle\right)_{1 \leq i \leq q}$ is of order $(1 \times q)$. From the Corollary 4 , if there exists $m_{0} \geq 1$ such that $\operatorname{rank} G_{m_{0}}^{T}=q$, then $\left(\Omega_{1}, g\right)$ is gradient efficient if and only if $\bigcap_{m \geq 1} \operatorname{ker}\left(M_{m} G_{m}^{T}\right)=\{0\}$. Then there exists $n_{1}, n_{2}, \ldots, n_{m}$ such that $n_{i} \neq n_{j}$ for $i \neq j$ and

$$
\bigcap_{i=1, m} \operatorname{ker}\left(M_{n_{i}} G_{n_{i}}^{T}\right)=\{0\}
$$

then $\left(\Omega_{1}, g\right)$ is gradient efficient. In particular if $m=q$, the condition (12) is equivalent to

$$
\left|\begin{array}{cc}
\sum_{j=1}^{r_{n_{1}}} \sum_{k=1}^{n}\left\langle g, w_{n_{1} j}\right\rangle\left\langle h_{1}, \frac{\partial w_{n_{1} j}}{\partial x_{k}}\right\rangle \cdots & \sum_{j=1}^{r_{n_{1}}} \sum_{k=1}^{n}\left\langle g, w_{n_{1} j}\right\rangle\left\langle h_{q}, \frac{\partial w_{n_{1} j}}{\partial x_{k}}\right\rangle \\
\vdots & \vdots \\
\sum_{j=1}^{r_{n_{q}}} \sum_{k=1}^{n}\left\langle g, w_{n_{q} j}\right\rangle\left\langle h_{1}, \frac{\partial w_{n_{q} j}}{\partial x_{k}}\right\rangle \cdots & \sum_{j=1}^{r_{n_{q}}} \sum_{k=1}^{n}\left\langle g, w_{n_{q j} j}\right\rangle\left\langle h_{q}, \frac{\partial w_{n_{q} j}}{\partial x_{k}}\right\rangle
\end{array}\right| \neq 0
$$




\section{GRADIENT REMEDIABILITY WITH MINIMUM ENERGY}

For $f \in L^{2}(0, T ; X)$, we study the existence and the unicity of an optimal control $u \in L^{2}\left(0, T ; I R^{P}\right)$ ensuring, at the time $T$, the gradient remediability of the disturbance $f$ such that $C \nabla H u+C \nabla F f=0$. That is the set defined by

$$
D=\left\{u \in L^{2}\left(0, T ; I R^{P}\right) / C \nabla H u+C \nabla F f=0\right\}
$$

is non empty.

We consider the function $J(u)=\|C \nabla H u+C \nabla F f\|_{I R^{q}}^{2}+\|u\|_{L^{2}\left(0, T ; I R^{P}\right)}^{2}$

The considered problem becomes $\min _{u \in D} J(u)$. For its resolution, we will use an extension of the Hilbert Uniqueness Methods (H.U.M). For $\theta \in I R^{q}$, let us note

$$
\|\theta\|_{*}=\left(\int_{0}^{T}\left\|B^{*} S^{*}(T-s) \nabla^{*} C^{*} \theta\right\|_{I R^{P}}^{2} d s\right)^{\frac{1}{2}}
$$

\|\|$_{*}$ is a semi-norm on $I R^{q}$. If the condition (7) is verified then it is a norm if and only if the system (1)-(2) is weakly gradient remediable on $[0, T]$. The corresponding inner product is given by $\langle\theta, \sigma\rangle_{*}=\int_{0}^{T}\left\langle B^{*} S^{*}(T-s) \nabla^{*} C^{*} \theta, B^{*} S^{*}(T-s) \nabla^{*} C^{*} \sigma\right\rangle d s$

and the operator $\Lambda: I R^{q} \rightarrow I R^{q}$ defined by

$$
\begin{aligned}
\Lambda \theta & =C \nabla H H^{*} \nabla^{*} C^{*} \theta \\
& =C \nabla \int_{0}^{T} S(T-s) B B^{*} S^{*}(T-s) \nabla^{*} C^{*} \theta d s
\end{aligned}
$$

Then, we have the following proposition:

\section{Proposition 6:}

If the condition (7) is verified, then \|\|$_{*}$ is a norm on $I R^{q}$ if and only if the system (1)-(2) is weakly gradient remediable on $[0, T]$ and the operator $\Lambda$ is invertible.

\section{Proof :}

We have 


$$
\begin{aligned}
& \|\theta\|_{*}=\left(\int_{0}^{T}\left\|B^{*} S^{*}(T-s) \nabla^{*} C^{*} \theta\right\|_{I R^{p}}^{2} d S\right)^{\frac{1}{2}}=0 \Rightarrow\left\|B^{*} S^{*}(T-s) \nabla^{*} C^{*} \theta\right\|_{L^{2}\left(0, T ; R^{P}\right)}=0 \\
& \Rightarrow B^{*} S^{*}(T-.) \nabla^{*} C^{*} \theta=0 \Rightarrow \theta \in \operatorname{ker}\left(B^{*} S^{*}(T-.) \nabla^{*} C^{*}\right)=\operatorname{ker}\left(B^{*} F^{*} \nabla^{*} C^{*}\right)
\end{aligned}
$$

But $\operatorname{ker}\left(B^{*} F^{*} \nabla^{*} C^{*}\right)=\bigcap_{m \geq 1} \operatorname{ker}\left(M_{m} f_{m}\right)$ (see the Proof of Proposition 5) and we have also $\bigcap_{m \geq 1} \operatorname{ker}\left(M_{m} G_{m}^{T}\right)=\bigcap_{m \geq 1} \operatorname{ker}\left(M_{m} f_{m}\right) \quad$ (see the Proof of Corollary 4)

then, $\operatorname{ker}\left(B^{*} F^{*} \nabla^{*} C^{*}\right)=\bigcap_{m \geq 1} \operatorname{ker}\left(M_{m} G_{m}^{T}\right)$ this gives $\theta \in \bigcap_{m \geq 1} \operatorname{ker}\left(M_{m} G_{m}^{T}\right)$ and since the Corollary 4 we obtain the result.

On the other hand the operator $\Lambda$ is symmetric, indeed

$$
\langle\Lambda \theta, \sigma\rangle_{I R^{q}}=\left\langle C \nabla H H^{*} \nabla^{*} C^{*} \theta, \sigma\right\rangle_{I R^{q}}=\left\langle\theta, C \nabla H H^{*} \nabla^{*} C^{*} \sigma\right\rangle_{I^{q}}=\langle\theta, \Lambda \sigma\rangle_{I R^{q}}
$$

and positive definite, indeed

$$
\begin{aligned}
\langle\Lambda \theta, \theta\rangle_{I R^{q}} & =\left\langle C \nabla H H^{*} \nabla^{*} C^{*} \theta, \theta\right\rangle_{I R^{q}} \\
& =\left\langle H^{*} \nabla^{*} C^{*} \theta, H^{*} \nabla^{*} C^{*} \theta\right\rangle_{L^{2}\left(0, T ; R^{P}\right)} \\
& =\int_{0}^{T}\left\langle B^{*} S^{*}(T-s) \nabla^{*} C^{*} \theta, B^{*} S^{*}(T-s) \nabla^{*} C^{*} \theta\right\rangle_{I R^{P}} d s \\
& =\|\theta\|_{*}^{2}>0, \quad \text { for } \theta \neq 0
\end{aligned}
$$

and then $\Lambda$ is invertible.

We give hereafter the expression of the optimal control ensuring the gradient remediability of a disturbance $f$ at the time $T$.

\section{Proposition 7:}

For $f \in L^{2}(0, T ; X)$, there exists a unique $\theta_{f} \in I R^{q}$ such that $\Lambda \theta_{f}=-C \nabla F f$ and the control $u_{\theta_{f}}()=.B^{*} S^{*}(.) \nabla^{*} C^{*} \theta_{f}$ verifies $C \nabla H u_{\theta_{f}}+C \nabla F f=0$. Moreover, it is optimal and $\left\|u_{\theta_{f}}\right\|_{L^{2}\left(0, T ; I R^{P}\right)}=\left\|\theta_{f}\right\|_{*}$.

\section{Proof :}

From the Proposition 6, the operator $\Lambda$ is invertible then, for $f \in L^{2}(0, T ; X)$, there exists a unique $\theta_{f} \in I R^{q}$ such that 


$$
\Lambda \theta_{f}=-C \nabla F f
$$

and if we put $u_{\theta_{f}}()=.B^{*} S^{*}(.) \nabla^{*} C^{*} \theta_{f}$, we obtain

$$
\begin{aligned}
& \Lambda \theta_{f}=C \nabla \int_{0}^{T} S(T-S) B B^{*} S^{*}(T-s) \nabla^{*} C^{*} \theta_{f} d s=C \nabla H u_{\theta_{f}} \\
& \Rightarrow-C \nabla F f=C \nabla H u_{\theta_{f}} \Rightarrow C \nabla H u_{\theta_{f}}+C \nabla F f=0 .
\end{aligned}
$$

The set $D$ defined by (13) is closed, convex and not empty. For $u \in D$, we have $J(u)=\|u\|_{L^{2}\left(0, T ; I R^{P}\right)}^{2} \cdot J$ is strictly convex on $D$, and then has a unique minimum at $u^{*} \in D$, characterized by $\left\langle u^{*}, v-u^{*}\right\rangle_{L^{2}\left(0, T ; I R^{P}\right)} \geq 0 ; \quad \forall v \in D$.

For $v \in D$, we have

$$
\begin{gathered}
\left\langle u_{\theta_{f}}, v-u_{\theta_{f}}\right\rangle_{L^{2}\left(0, T ; I R^{P}\right)}=\left\langle B^{*} S^{*}(.) \nabla^{*} C^{*} \theta_{f}, v-B^{*} S^{*}(.) \nabla^{*} C^{*} \theta_{f}\right\rangle_{L^{2}\left(0, T ; I R^{P}\right)} \\
=\left\langle\theta_{f}, C \nabla H v-\Lambda \theta_{f}\right\rangle_{I R^{q}}=0
\end{gathered}
$$

Since $u^{*}$ is unique, then $u^{*}=u_{\theta_{f}}$ and $u_{\theta_{f}}$ is optimal with

$$
\left\|u_{\theta_{f}}\right\|_{L^{2}\left(0, T ; I R^{P}\right)}^{2}=\left\|B^{*} S^{*}(.) \nabla^{*} C^{*} \theta_{f}\right\|_{L^{2}\left(0, T ; I R^{P}\right)}^{2}=\left\|\theta_{f}\right\|_{*}^{2} .
$$

\section{APPROXIMATIONS AND NUMERICAL SIMULATIONS}

This section concerns approximations and numerical simulations of the problem of gradient remediability. First we give an approximation of $\theta_{f}$ as a solution of a finite dimension linear system $A x=b$ and then the optimal control $u_{\theta_{f}}$, with a comparison between the corresponding observation noted $z_{u, f}$ and the normal case.

\subsection{Approximations}

- Coefficients of the system: For $i, j \geq 1$, let

$a_{i j}=\left\langle\Lambda e_{i}, e_{j}\right\rangle_{I R^{q}}$ where $\left(e_{i}\right)_{1 \leq i \leq q}$ is the canonical basis of $I R^{q}$, we have

$\Lambda e_{i}=C \nabla \int_{0}^{T} S(T-s) B B^{*} S^{*}(T-s) \nabla^{*} C^{*} e_{i} d s$

And for $M, N$ sufficiently large, we have 
$a_{i j} \approx \sum_{m=1}^{M} \sum_{l=1}^{r_{m}} \sum_{m^{\prime}=1}^{N} \sum_{h=1}^{r_{m^{\prime}}} \sum_{\tau=1}^{p}\left(\frac{e^{\left(\lambda_{m}+\lambda_{m^{\prime}}\right) T}-1}{\lambda_{m}+\lambda_{m^{\prime}}}\right)\left\langle g_{\tau}, w_{m l}\right\rangle_{L^{2}\left(\Omega_{\tau}\right)}\left\langle g_{\tau}, w_{m^{\prime} h}\right\rangle_{L^{2}\left(\Omega_{\tau}\right)} \sum_{k^{\prime}=1}^{n}\left\langle\frac{\partial w_{m^{\prime} h}}{\partial x_{k^{\prime}}}, h_{i}\right\rangle_{L^{2}\left(D_{i}\right)} \sum_{k=1}^{n}\left\langle\frac{\partial w_{m l}}{\partial x_{k}}, h_{j}\right\rangle_{L^{2}\left(D_{j}\right)}$

and $\Lambda \theta_{f}=-C \nabla F f$

$b_{j}=-\left\langle C \nabla F f, e_{j}\right\rangle_{I R} q$

For $N$ sufficiently large, we have

$b_{j} \approx-\sum_{m^{\prime}=1}^{N} \sum_{h=1}^{r_{m^{\prime}}} \sum_{k=1}^{n}\left\langle\frac{\partial w_{m^{\prime} h}}{\partial x_{k}}, h_{j}\right\rangle_{L^{2}\left(D_{j}\right)} \int_{0}^{T} e^{\lambda_{m^{\prime}}(T-s)}\left\langle f(s), w_{m^{\prime} h}\right\rangle_{L^{2}(\Omega)} d s$

- The optimal control: In this part, we give an

approximation of the optimal control $u_{\theta_{f}}$ which is defined by $u_{\theta_{f}}()=.B^{*} S^{*}(T-.) \nabla^{*} C^{*} \theta_{f}$.

Its function coordinates $u_{j, \theta_{f}}($.$) are given by$

$u_{j, \theta_{f}}()=.\left\langle g_{j}, S^{*}(T-.) \nabla^{*} C^{*} \theta_{f}\right\rangle \approx \sum_{m^{\prime}=1}^{N} \sum_{h=1}^{r_{m}{ }^{\prime}} \sum_{k=1}^{n} \sum_{i=1}^{q} \theta_{i, f} e^{\lambda_{m^{\prime}(T-.)}}\left\langle g_{j}, w_{m^{\prime} h}\right\rangle_{L^{2}\left(\Omega_{j}\right)}\left\langle\frac{\partial w_{m^{\prime} h}}{\partial x_{k}}, h_{i}\right\rangle$

for a large integer $N$.

- Cost: The minimum energy (cost) is defined by

$\left\|u_{\theta_{f}}\right\|_{L^{2}\left(0, T ; I R^{p}\right)}=\left(\int_{0}^{T}\left\|B^{*} S^{*}(T-s) \nabla^{*} C^{*} \theta_{f}\right\|_{I R}^{2} d s\right)^{\frac{1}{2}}$
$\approx\left(\sum_{j=1}^{p} \int_{0}^{T}\left(\sum_{m^{\prime}=1}^{N} \sum_{h=1}^{r_{m^{\prime}}} \sum_{k=1}^{n} \sum_{i=1}^{q} e^{\lambda_{m}(T-s)} \theta_{i, f}\left\langle h_{i}, \frac{\partial w_{m^{\prime} h}}{\partial x_{k}}\right\rangle\left\langle g_{j}, w_{m^{\prime} h}\right\rangle\right)^{2} d s\right)^{\frac{1}{2}}$

for $N$ sufficiently large.

- The corresponding observation: The observation corresponding to the control is given by

$$
z_{u_{\theta_{f}}, f}(t)=C \nabla S(t) y^{0}+C \nabla \int_{0}^{t} S(t-s) B u_{\theta_{f}}(s) d s+C \nabla \int_{0}^{t} S(t-s) f(s) d s
$$

Its coordinates $\left(z_{j, u_{f}, f}(.)\right)_{1 \leq j \leq q}$ are obtained for a large integer $N$, as follows:

$$
\begin{aligned}
& z_{j, u_{\theta_{f}}, f}(t) \approx \sum_{m^{\prime}=1}^{N} \sum_{h=1}^{r_{m^{\prime}}} \sum_{k=1}^{n} e^{\lambda_{m^{\prime}} t}\left\langle y^{0}, w_{m^{\prime} h}\right\rangle_{L^{2}(\Omega)}\left\langle h_{j}, \frac{\partial w_{m^{\prime} h}}{\partial x_{k}}\right\rangle_{L^{2}\left(D_{j}\right)}+ \\
& \sum_{m^{\prime}=1}^{N} \sum_{h=1}^{r_{m^{\prime}}} \sum_{k=1}^{n} \sum_{i=1}^{p}\left\langle g_{i}, w_{m^{\prime} h}\right\rangle\left\langle\frac{\partial w_{m^{\prime} h}}{\partial x_{k}}, h_{j}\right\rangle \int_{0}^{t} e^{\lambda_{m^{\prime}}(t-s)} u_{i, \theta_{f}}(s) d s+\sum_{m^{\prime}=1}^{N} \sum_{h=1}^{r_{m^{\prime}}} \sum_{k=1}^{n}\left\langle\frac{\partial w_{m^{\prime} h}}{\partial x_{k}}, h_{j}\right\rangle \int_{0}^{t} e^{\lambda_{m^{\prime}}(t-s)}\left\langle f(s), w_{m^{\prime} h}\right\rangle d s
\end{aligned}
$$




\subsection{Numerical simulations}

We consider without loss of generally the following diffusion system

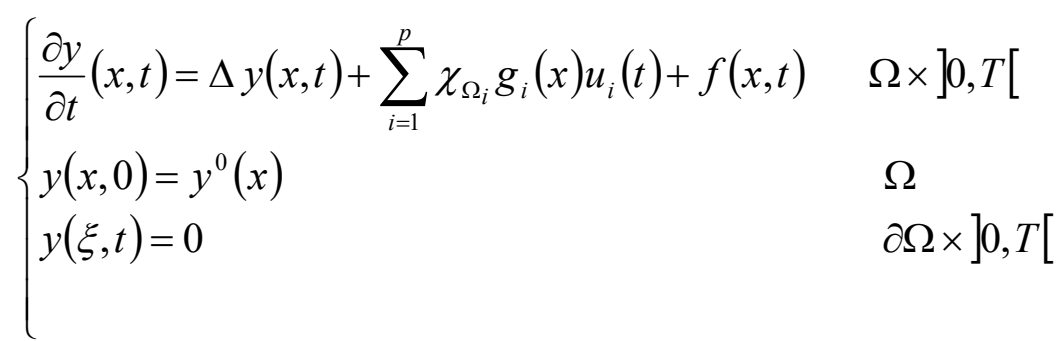

with $\Omega=] 0,1\left[\right.$ and a Dirichlet boundary condition. In this case, the functions $w_{m}($.$) are$ defined by $w_{m}(x)=\sqrt{2} \sin (m \pi x) ; m \geq 1$. The associated eigenvalues are simple and given by $\lambda_{m}=-m^{2} \pi^{2} ; \quad m \geq 1$. Then in the case of:

- an initial state: $y^{0}()=$.0 ,

- a sensor: $(D, h)$ with $D=] 0,1\left[\right.$ and $h(x)=\sqrt{2} x^{2} \quad(q=1)$

- an efficient actuator: $(\Omega, g)$ with $\Omega=] 0,1\left[\right.$ and $g(x)=2 x^{3}(p=1)$

- a disturbance function: defined by $f(x, t)=240 e^{-\left(\frac{t}{10}+x\right)} ; t>0$

For $M=N=1$ and $T=70$, we obtain numerical results illustrating the theoretical results established in previous sections. Hence, in figure1, we give the representations of the discrete observation $z_{u, f}$ corresponding to the control $u=u_{\theta_{f}}$ and the disturbance $f$ and $z_{0,0}$ which represent the normal observation, that is $u=0$ and $f=0$.

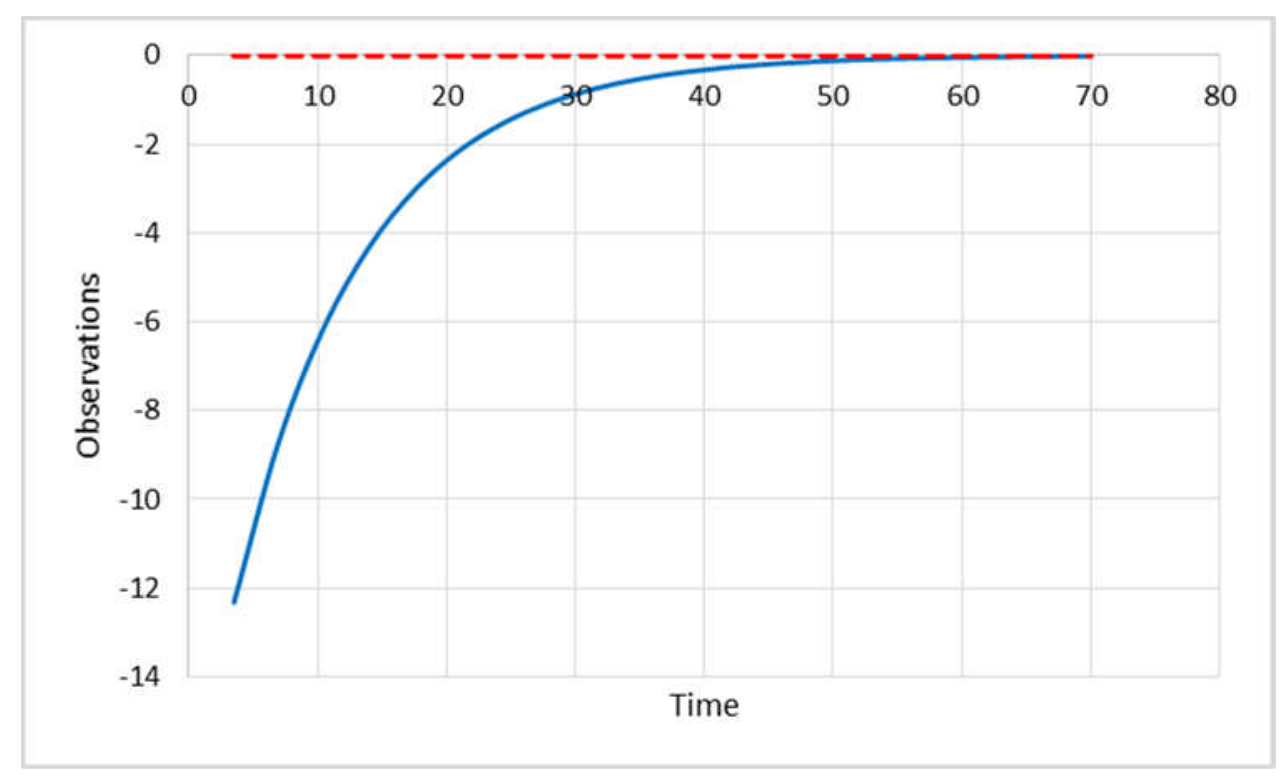

Fig.1. Representation of $z_{u, f}$ (blue line) and $z_{0,0}$ (red line). 
This figure show that for $t$ sufficiently large $(t \geq 50)$, the disturbance $f$ is compensate by the control optimal $u_{\theta_{f}}$ at the time $T(T=70)$ that is, we have $z_{u_{\theta_{f}}, f}(t) \equiv z_{0,0}(t)$.

The optimal control $u_{\theta_{f}}$ ensuring the gradient remediability of the disturbance $f$, is represented in figure 2.

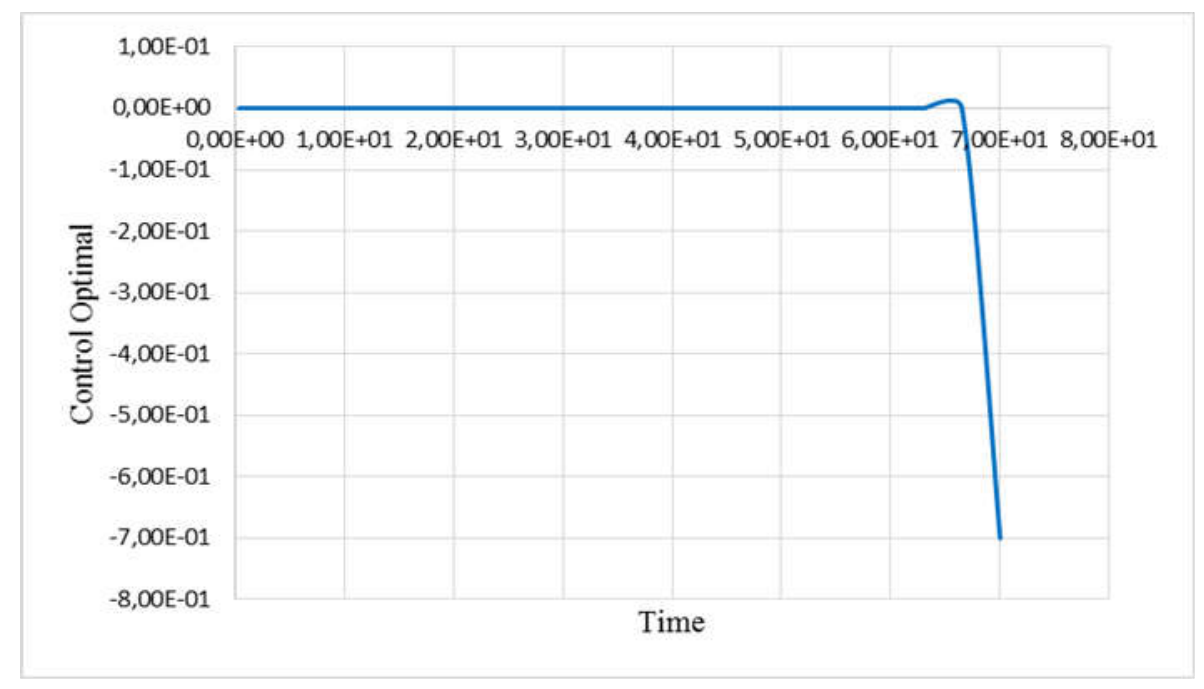

Fig.2. Representation of the optimal control $u_{\theta_{f}}$ (blue line)

\section{CONCLUSION}

In this paper, which is an extension of previous works to the analysis of the gradient of a large class of parabolic systems, new notions of weak and exact gradient remediability are introduced and characterized. The relation between the notion of gradient remediability and the notion of gradient controllability is also studied. We have shown that a parabolic system is gradient remediable if it is gradient controllable. Furthermore, we have shown that the exact and weak gradient remediability of a system may depend on the structure and the number of the actuators and sensors. Using an extension of the Hilbert Uniqueness Method, we have shown how to find the optimal control ensuring the gradient remediability of the known or unknown disturbance. The results of illustrative examples and numerical approximations are acceptable.

These results are developed for a class of discrete linear distributed parabolic systems, but the considered approach can be extended to other class of systems with a convenient choice of space. 


\section{REFERENCES}

[1] Curtain R.F, and Zwart H, An introduction to infinite dimensional linear systems theory, in Applied Mathematics, Springer-Verlag, 1995.

[2] Lions J.L, Contrôlabilité exacte. Perturbation et stabilisation des systems distribués, Tome 1. Masson, 1988.

[3] Berrahmoun L, Localisation d'actionneur pour la Contrôlabilité de Systèmes Paraboliques et Hyperboliques. Application par Dualité à la Localisation de Capteurs, Ph.D dissertation, University, Mohammed V, Rabat, April 1984.

[4] El jai A, Sensors and actuators in distributed systems, International Conference on control and identification of distributed systems. Vorau, Autriche, July 1986.

[5] El jai A, and El Yacoubi S, On the number of actuators in distributed system, International Journal, Applied Mathematics and Computer Science, 1993, 3(4), 1993.

[6] El jai A, Simon M.C, and Zerrik E, Regional observability and sensor structures, International journal on sensors and actuators, 1993, 39(2), 95-102.

[7] Zerrik E, Benhadid S, and Bourray H, Sensors and regional observability of the wave equation, Int. Journal on Sensors and Actuators, 2007, 313-328.

[8] Zerrik E, Bourray H, and Benhadid S, Sensors and boundary state reconstruction of hyperbolic systems, International Journal of Applied Mathematics and Computer Science, 2010, 20(2), 227-238.

[9] Zerrik E, Boutoulout A, and Kamal A, Regional gradient controllability of parabolic systems, Appl. Math. And Comp. Sci, 1999, 9(4), 101-121.

[10] Alsaphory R.A, Al-jawari N, and Al-qaisi I, Regional Gradient Detectability for Infinite Dimensional Systems, Tikrit Journal of Pure Science, 2010, 15(2), 1813-1662.

[11] Benhadid S, Rekkab S, and Zerrik E, Sensors and boundary gradient observability of hyperbolic systems, International Control and Automation, 2012, 3(1), 78-89.

[12] Benhadid S, Rekkab S, and Zerrik E, Sensors and regional gradient observability of hyperbolic systems, International Journal of Computers \& Technology, 2013, 4(3), 295-316.

[13]Zerrik E, and Bourray H, Regional gradient observability for parabolic systems, International Journal of Applied Mathematics and Computer Science, 2003, 13, 139-150.

[14] Afifi L, Chafiai A, and El jai A, Compensation Spatial en Temps Fini dans les Systèmes 
Distribués, L.T.S, Université de Perpignant, Rapport Interne, 1998, (1498).

[15] Afifi L, Magri E.M, and El jai A, Compensation Problem in Finite Linear Dynamical Systems, Applied Mathematical Sciences, 2008, 2(49), 2219-2238.

[16] Afifi L, Chafiai A, and El jai A, Spatial Compensation of Boundary Disturbances by Boundary Actuators, Int. J. Appl. Math. Comput. Sci., 2001, 11(4), 899-920.

[17] Lions J.L, Optimal control systems governed by partial differential differential equations, Springer-Verlag, New York, 1971.

[18] Trenoguine V, Analyse fonctionnelle, Edition Mir, Moscou, 1985.

[19] El jai A, and Pritchard A.J, Sensors and actuators in the analysis of distributed systems, Ellis Horwood, Series in Applied Mathematics, J. wiley, 1988.

[20] Ipsen I.C.F, Numerical Matrix Analysis: Linear Systems and Least Squares, Society for Industrial and Applied Mathematics, Philadelphia, 2009.

\section{How to cite this article:}

Rekkab S, Benhadid S. Gradient remadiability in linear distributed parabolic systems:

Analysis, approximations and simulations. J. Fundam. Appl. Sci., 2017, 9(3), 1535-1558. 